\title{
Hereditary hemochromatosis: Is the gene race over?
}

\author{
Paolo Gasparini ${ }^{1}$ and Clara Camaschella ${ }^{2}$
}

\author{
${ }^{1}$ Genetica Medica, Dipartimento di Patologia Generale, Seconda Università di Napoli e TIGEM, Napoli, Italy; \\ ${ }^{2}$ Dipartimento di Scienze Cliniche e Biologiche, Università di Torino, Azienda Ospedaliera San Luigi, Orbassano, \\ Torino, Italy
}

European Journal of Human Genetics (2004) 12, 341-342. doi:10.1038/sj.ejhg.5201176

The fascinating race to understand the genetic basis of hereditary hemochromatosis (HFE) is over. Hemochromatosis (OMIM *235200) is a common autosomal recessive disease with an estimated prevalence of 2-5/1.000 in Caucasians and a corresponding carrier frequency of 0.045-0.071. Clinical complications, due to chronic dietary iron overload, usually occur in middle life and include liver cirrhosis, diabetes, hypermelanotic pigmentation of the skin, hypogonadism, heart failure and increased susceptibility to hepatocellular carcinoma. The disease is due to disruption of the molecular mechanisms that tightly restrict iron absorption in the gut and maintain iron homeostasis. Almost 30 years ago, Simon et al ${ }^{1}$ showed that the disease segregates with the major histocompatibility complex and described the disequilibrium with the HLA serotype A3, thus defining one of the first loci ever mapped for human inherited diseases. A novel HLA class I-like gene, named HFE, was isolated 20 years later and a common mutation (C282Y) described in the majority of the affected cases. ${ }^{2}$ This mutation disrupts a putative $\beta_{2}$-microglobulin binding site, and reduces the binding of HFE to transferrin receptor (TFR) on the cell membrane. Initially, hemochromatosis was considered a homogenous genetic disorder, but the finding of several affected chromosomes negative for HFE mutations and the identification of families not linked to HFE prompted the search of additional genetic loci and genes.

In 1999, a locus for juvenile hemochromatosis (HFE2, OMIM \#602390), a form characterized by a severe iron overload and early onset of cardiomyopathy, diabetes and hypogonadism, was mapped on the long arm of chromosome $1 .^{3}$ In 2000, a new hemochromatosis locus was identified on 7q22 (HFE3, OMIM \#604250) and causative mutations detected within transferrin receptor 2 (TFR2) gene. ${ }^{4}$ TFR2 encodes a transmembrane protein with a significant homology to TFR, is highly expressed in the liver and, as TFR, it binds transferrin, although at lower affinity. Its function remains a mystery.
Families with a rare form of autosomal-dominant hemochromatosis not linked to HFE (HFE4, OMIM \#606069), characterized by early iron accumulation in reticuloendothelial cells, and presenting mutations in the SLC4OA1 gene on chromosome $2 \mathrm{q}$ were then described. ${ }^{5}$ SCL4OA1 encodes ferroportin 1 (FPN1), a transmembrane protein with a critical role in iron export in enterocytes, hepatocytes and macrophages.

Strong efforts to clone the HFE2 gene from the difficult, partially covered and pericentromeric $1 \mathrm{q}$ region have been carried out during the past 5 years. During these studies mutations in the antimicrobial peptide hepcidin (HAMP; OMIM 606464) gene have been detected in a subgroup of patients affected by juvenile hemochromatosis not linked to HFE2. ${ }^{6}$ There is strong evidence that hepcidin is central to iron homeostasis in mice. Hamp inactivation in USF2 knock out mice cause severe iron overload, whereas Hamp hyperexpression in transgenic mice causes severe iron deficiency barely compatible with survival. ${ }^{7}$ The discovery that mutations of hepcidin cause juvenile hemochromatosis reinforced the concept of hepcidin as the key regulator of iron absorption in mammals.

Now with the recent publication by Papanikolau et al, ${ }^{8}$ a last piece has been added to complete the puzzle of hemochromatosis genes. This novel gene (HJV, OMIM 608374) encodes for a protein named hemojuvelin, which, as hepcidin, must be crucial to iron metabolism. Its transcript expression is restricted to liver, heart and skeletal muscle, similar to that of HAMP. The 426 amino-acid protein shows homology to $R G M$, a guidance molecule of the retinotectal system, has a signal peptide, a large von Willebrand D-like domain and a C-terminal transmembrane domain ${ }^{8}$ with a likely GPI anchor. It is still too early to understand the hemojuvelin function, but interestingly, hepcidin urinary levels are depressed in these patients, ${ }^{8}$ suggesting that hemojuvelin is a modulator of HAMP expression. Furthermore, these findings and the pattern of 


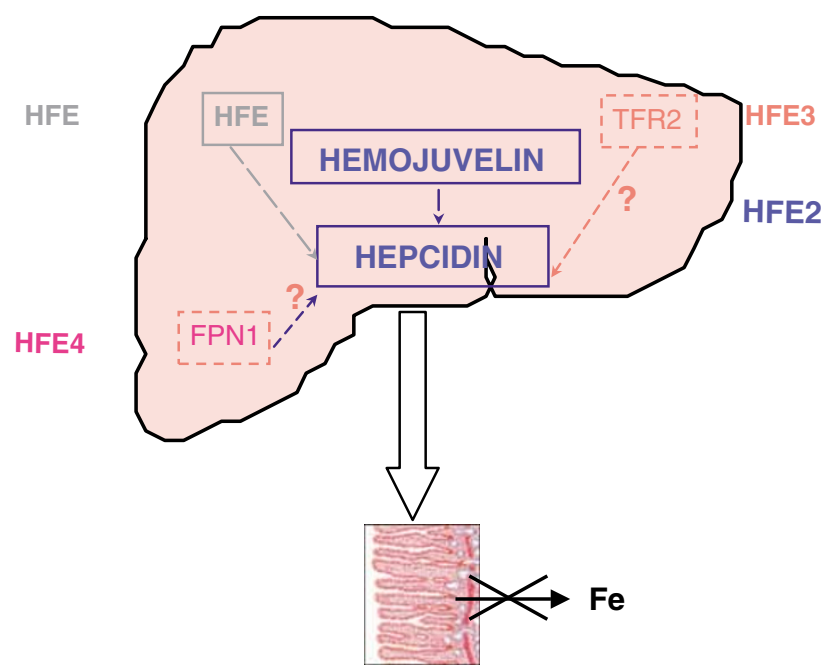

Figure 1 The central role of hepcidin in iron absorption and hemochromatosis. The network of the hemochromatosis proteins in the liver is shown. The hepcidin effect on intestinal iron absorption is indicated by the large arrow. Genetic or functional relationship's of HFE and hemojuvelin to hepcidin are indicated by the dotted arrows. Question mark over the arrows indicates unproven relationships. The type of hemochromatosis caused by mutations in the cognate genes are indicated outside the liver using identical color.

mRNA expression exclude that hemojuvelin is the hypothesized, though still elusive, hepcidin receptor.

From the available data, it seems that a minority of juvenile hemochromatosis patients have an $H A M P$-related disorder, whereas most have mutations in the HJV gene. ${ }^{9}$ These findings strongly suggest that our knowledge of the genetic bases of hemochromatosis is complete.
All hemochromatosis genes are highly expressed in the liver (Figure 1). Hepcidin inactivation by HAMP or $H J V$ mutations causes the most severe form of hemochromatosis (HFE2). Hepcidin mRNA is inappropriately low compared to iron loading in HFE-hemochromatosis, both in mouse models and in patients, ${ }^{10}$ suggesting that HFE might regulate hepcidin. Digenic inheritance, recently reported for iron-loaded patients who are heterozygous for both HFE and HAMP mutations, ${ }^{11}$ is in agreement with this concept and suggests some kind of interaction between HFE and hepcidin. It is still unknown whether HFE3 (and HFE4, although this is an atypical form) have low hepcidin expression, but we speculate that all genetic iron overload converge into a final hepcidin-dependent pathway (Figure 1).

Now that all the players in the game of iron absorption regulation have been discovered, the research challenge is to understand the specific role of their cognate proteins and their relationships in maintaining iron homeostasis.

\footnotetext{
References

1 Simon M et al: Gut 1976; 17: 332-334.

2 Feder JN et al: Nat Genet 1996; 13: 399-408.

3 Roetto A et al: Am J Hum Genet 1999; 64: 1388-1393.

4 Camaschella C et al: Nat Genet 2000; 25: 14-15.

5 Montosi G et al: J Clin Invest 2001; 108: 619-623.

6 Roetto A et al: Nat Genet 2003; 33: 21-22.

7 Ganz T: Blood 2003; 102: 783-788.

8 Papanikolaou G et al: Nat Genet 2004; 36: 77-82.

9 Lanzara et al: The spectrum of hemojuvelin gene mutations in sqlinked juvenile hemochromatosis. Blood, in press.

10 Bridle KR et al: Lancet 2003; 361: 669-673.

11 Merryweather-Clarke A et al: Hum Mol Genet 2003; 12: 2241-2247.
} 